\begin{tabular}{|c|l|}
\hline Title & Diamagnetic Inequalities for Systems of Nonrelativistic Particles with a Quantized Field \\
\hline Author(s) & Hiroshima, F. \\
\hline Citation & Hokkaido University Preprint Series in Mathematics, 297, 1-23 \\
\hline Issue Date & 1995-6-1 \\
\hline DOI & 10.14943/83444 \\
\hline Doc URL & http://hdl.handle.net/2115/69048 \\
\hline Type & bulletin (article) \\
\hline File Information & pre297.pdf \\
\hline
\end{tabular}

Instructions for use 
Diamagnetic Inequalities for Systems of Nonrelativistic Particles with a Quantized Field

\author{
Fumio Hiroshima
}

Series $\sharp 297$. June 1995 


\section{HOKKAIDO UNIVERSITY \\ PREPRINT SERIES IN MATHEMATICS}

$\sharp 273$ K. Tsutaya, Global existence of small amplitude solutions for the Klein-Gordon-Zakharov equations, 11 pages. 1994.

$\sharp 274$ H. Kubo, On the critical decay and power for semilinear wave equations in odd space dimensions, 22 pages. 1994.

$\sharp 275$ N. Terai, T. Hibi, Alexander duality theorem and second Betti numbers of Stanley-Reisner rings, 2 pages. 1995.

$\sharp 276$ N. Terai, T. Hibi, Stanley-Reisner rings whose Betti numbers are independent of the base field, 12 pages. 1995.

$\sharp 277 \quad$ N. Terai, T. Hibi, Computation of Betti numbers of monomial ideals associated with cyclic polytopes, 11 pages. 1995.

$\sharp 278$ N. Terai, T. Hibi, Computation of Betti numbers of monomial ideals associated with stacked polytopes, 8 pages. 1995.

$\sharp 279$ N. Terai, T. Hibi, Finite free resolutions and 1-skeletons of simplicial $(d-1)$-spheres, 3 pages. 1995.

$\sharp 280$ N. Terai, T. Hibi, Monomial ideals and minimal non-faces of Cohen-Macanlay complexes, 6 pages. 1995.

$\sharp 281$ A. Arai, N. Tominaga, Analysis of a family of strongly commuting self-adjoint operators with applications to perturbed d'Alembertians and the external field problem in quantum field theory, 44 pages. 1995.

$\sharp 282$ T. Mikami, Asymptotic behavior of the first exit time of randomly perturbed dynamical systems with a repulsive equilibrium point, 29 pages. 1995.

\#283 K. Iwata, J. Schäfer, Markov property and cokernels of local operators, 17 pages. 1995.

\#284 T. Nakazi, M. Yamada, Riesz's Functions In Weighted Hardy And Bergman Spaces, 20 pages. 1995.

$\sharp 285$ K. Hidano, K. Tsutaya, Scattering theory for nonlinear wave equations in the invariant Sobolev space, 32 pages. 1995.

$\sharp 286$ A. Arai, Strong coupling limit of the zero-energy-state density of the Dirac-Weyl operator with a singular vector potential, 8 pages. 1995 .

\#287 T. Nakazi, Factorizations of outer functions and extremal problems, 15 pages. 1995.

$\sharp 288$ A. Kishimoto, The Rohlin property for automorphisms of UHF algebras, 15 pages. 1995.

\#289 K. Goto, A. Yamaguchi and I. Tsuda, Nine-bit states cellular automata are capable of simulating the pattern dynamics of coupled map lattice, 24 pages. 1995.

\#290 Y. Giga, Interior derivative blow-up for quasilinear parabolic equations, 16 pages. 1995.

$\sharp 291$ F. Hiroshima, Functional Integral Representation of a Model in QED, 48 pages. 1995.

$\sharp 292$ N. Kawazumi, A Generalization of the Morita-Mumford Classes to Extended Mapping Class Groups for Surfaces, 11 pages. 1995.

$\sharp 293$ P. Aviles and Y. Giga, The distance function and defect energy, 23 pages. 1995.

$\sharp 294$ S. Izumiya and A. Takiyama, A time-like surface in Minkowski 3-space which contains pseudocircles, 11 pages. 1995.

$\sharp 295$ S. Izumiya, Local classifications of multi-valued solutions of quasilinear first order partial differential equations, 12 pages. 1995.

$\sharp 296$ A. Kishimoto, A Rohlin property for one-parameter automorphism groups, 27 pages. 1995. 


\title{
Diamagnetic Inequalities for Systems of Nonrelativistic Particles with a Quantized Field
}

\author{
Fumio Hiroshima \\ Department of Mathematics, Hokkaido University \\ Sapporo 060, Japan
}

\begin{abstract}
By unitary transformations (gauge transformation, Bogoliubov transformation) and the strong Trotter product formula, diamagnetic inequalities for the Pauli-Fierz model of QED and the Nelson model are derived. In the Nelson model, the unitary transformation defines effective potentials. Moreover, the infimum of the spectrum of Hamiltonians for these models are estimated and some abstract Kato's inequalities are obtained.
\end{abstract}




\section{INTRODUCTION}

The purpose of this paper is to derive diamagnetic inequalities for the both of the Pauli-Fierz model of $\mathrm{QED}([1])$ and the Nelson model([2]).

In classical models, diamagnetic inequalities for the Hamiltonian

$$
\mathbf{H}_{c l}=\frac{1}{2} \sum_{\mu=1}^{d}\left(-i D_{\mu}-A_{\mu}\right)^{2}
$$

are given as follows $([3])$ :

$$
\begin{gathered}
\left|\left(f, e^{-t\left(\mathbf{H}_{c l} \dot{+} V_{+}-V_{-}\right)} g\right)_{L^{2}\left(\mathbb{R}^{d}\right)}\right| \leq \underset{f}{f, g \in L^{2}\left(\mathbb{R}^{d}\right), t \geq 0} \\
\left(|f|, e^{-t\left(-\frac{1}{2} \Delta+V_{+}-V_{-}\right)}|g|\right)_{L^{2}\left(\mathbb{R}^{d}\right)}
\end{gathered}
$$

where $A_{\mu} \in L_{l o c}^{2}\left(\mathbb{R}^{d}\right)$ is a magnetic vector potential, $\Delta$ denotes the d-dimensional Laplacian and $V_{+}$(resp. $-V_{-}$) are the nonnegative (resp.nonpositive) part of a scalar potential $V$. The diamagnetic inequalities (1.1) can be derived by applying the strong Trotter product formula and gauge transformations([3]). Also path integral representations of the heat semigroup with the infinitesimal generator $\mathbf{H}_{c l}$ gives (1.1)([4]). Moreover from (1.1) one can easily derive abstract kato's inequalities $([5,6,7])$;

$$
\begin{aligned}
& \left(\left(\mathbf{H}_{c l} \dot{+} V_{+} \dot{-} V_{-}\right)^{\frac{1}{2}} \phi,\left(\mathbf{H}_{c l} \dot{+} V_{+} \dot{-} V_{-}\right)^{\frac{1}{2}}|\psi|\right)_{L^{2}\left(\mathbb{R}^{d}\right)} \leq \Re\left((\operatorname{sign} \psi) \phi,\left(\mathbf{H}_{c l} \dot{+} V_{+} \dot{-} V_{-}\right) \psi\right)_{L^{2}\left(\mathbb{R}^{d l}\right)}, \\
& \psi \in D\left(\left(\mathbf{H}_{c l} \dot{+} V_{+} \dot{-} V_{-}\right)\right), \phi \geq 0, \phi \in D\left(\left(\mathbf{H}_{c l} \dot{+} V_{+} \dot{-} V_{-}\right)^{\frac{1}{2}}\right) .
\end{aligned}
$$

In PART I, we discuss the Pauli-Fierz model of QED. The Pauli-Fierz model of QED describes a quantum system of nonrelativistic charged particles (electrons) interacting with a quantized radiation field. The Hamiltonian of the Pauli-Fierz model with an ultraviolet cutoff is defined as an operator acting in the Hilbert space $\mathcal{M}=L^{2}\left(\mathbb{P}^{d}\right) \otimes \mathcal{F}(\mathcal{W})$ as follows:

$$
\mathbf{H}_{\rho}^{P}=\frac{1}{2} \sum_{\mu=1}^{d}\left(-i D_{\mu} \otimes I-A_{\mu}(\rho)\right)^{2}+I \otimes \mathbf{H}_{0}^{P}
$$


where $\mathcal{F}(\mathcal{W})$ is the Boson Fock space over $\mathcal{W}=\underbrace{L^{2}\left(\mathbb{R}^{d}\right) \oplus \ldots \oplus L^{2}\left(\mathbb{P}^{d}\right)}_{d-1}, \mathbf{H}_{0}^{P}$ denotes the free Hamiltonian of the quantized radiation field and $A_{\mu}(\rho)$ is the time-zero radiation field in the $\mu$-th direction with an ultraviolet cut-off function $\rho$. This model has been studied by many authors $([8,9,10,11,12,13,14]$ and references therein $)$. In [12], under some conditions for $\rho$ and $V$, functional integral representations of the heat semigroup, the infinitesimal generator of which is a self-adjoint extension of $\mathbf{H}_{\rho}^{P} \dot{+} V_{+} \dot{-} V_{-}$restricted to some domain, has been derived. Moreover, the following diamagnetic inequality was found;

$$
\begin{aligned}
\left|\left(F, e^{-t\left(\mathbf{H}_{\rho}^{P} \dot{+} V_{+} \dot{-} V_{-}\right)} G\right)_{\mathcal{M}}\right| \leq & \left(\|F\|_{\mathcal{F}(\mathcal{W})}, e^{-t\left(-\frac{1}{2} \Delta \dot{+} V_{+}-V_{-}\right)}\|G\|_{\mathcal{F}(\mathcal{W})}\right)_{L^{2}\left(\mathbb{R}^{d}\right)} \\
& F, G \in \mathcal{M}, t \geq 0 .
\end{aligned}
$$

In PART I, from the operator-theoretical point of view, by using gauge transformations, we derive (1.3) under a condition for $\rho$ different from that in [12].

In PART II, we discuss the Nelson model. The Nelson model describes a quantum system of nonrelativistic particles (nucleons) interacting with a scalar field (meson field) $([15,16])$.

As for the Pauli-Fierz mode we get (1.3), for the Nelson model, using Bogoliubov transformations $([17])$, which defines effective potentials, we also get such inequalities. The Hamiltonian of the Nelson model is defined as an operator acting in the Hilbert space $\mathcal{N}=L^{2}\left(\mathbb{R}^{d N}\right) \otimes \mathcal{F}$ as follows:

$$
\mathbf{H}_{\rho}^{N}=-\frac{1}{2} \sum_{j=1}^{N} \Delta_{j} \otimes I-g \mathbf{H}_{I}(\rho)+I \otimes \mathbf{H}_{0}^{N},
$$

where $\mathcal{F}\left(L^{2}\left(\mathbb{R}^{d}\right)\right)=\mathcal{F}$, the Boson Fock space over $L^{2}\left(\mathbb{R}^{d}\right), \mathbf{H}_{0}^{N}$ denotes the free Hamiltonian of the scalar field, $\mathbf{H}_{I}(\rho)$ is the time-zero field with an ultraviolet cut-off function $\rho$ and $g$ is a coupling constant. The main theorem of PART II is to derive the following inequalities:

$$
\begin{aligned}
\left|\left(F, e^{-t\left(\mathbf{H}_{\rho}^{N} \dot{+} V_{+}-V_{-}\right)} G\right)_{\mathcal{N}}\right| \leq & \left(\|F\|_{\mathcal{F}}, e^{-t\left(-\frac{1}{2} \sum_{j=1}^{N} \Delta_{j}+g V_{e f f} \dot{+} V_{+}-V_{-}\right)}\|G\|_{\mathcal{F}}\right)_{L^{2}\left(\mathbb{R}^{d N}\right)} \\
& F, G \in \mathcal{N}, t \geq 0
\end{aligned}
$$


where $V_{\text {eff }}$ is defined by (so called effective potential)

$$
V_{e f f}\left(x_{1}, \ldots, x_{N}, \rho\right)=-\frac{1}{2}\left\|\frac{\hat{\rho}}{\omega_{\mu}} \sum_{j=1}^{N} e^{-i \cdot x_{j}}\right\|_{L^{2}\left(\mathbb{R}^{d}\right)}^{2},
$$

where $\omega_{\mu}(k)=\sqrt{k^{2}+\mu^{2}}, \mu>0$. It is interesting for the effective potentials to appear in a. scaling limit of $\mathbf{H}_{\rho}^{N}([16])$.

In the both of PART I,II, we get some abstract Kato's inequalities similar to (1.2). The outline of this paper is as follows.

(PART I)

In subsection 2.1 , we find unitary transformations, $\mathcal{U}_{\mu}(\rho), \mu=1, \ldots, d$, on $\mathcal{M}$ (so called gauge transformation) such that

$$
\mathcal{U}_{\mu}(\rho)\left(-\frac{1}{2} \Delta_{\mu} \otimes I\right) \mathcal{U}_{\mu}(\rho)^{-1}=\frac{1}{2}\left(-i D_{\mu} \otimes I-A_{\mu}(\rho)\right)^{2}, \mu=1, \ldots, d
$$

and give a definition of $\mathbf{H}_{\rho}^{P}$ as a self-adjoint operator in $\mathcal{M}$. In subsection 2.2 , using the strong Trotter product formula and the unitary equivalence (1.5), we get (1.3). Furthermore, we obtain some inequalities from (1.3).

(PART II)

In subsection 3.1, we find a unitary transformation $\mathcal{U}(g \rho)$ (so called Bogoliubov transformation) such that

$$
\mathcal{U}(g \rho)\left(I \otimes \mathbf{H}_{0}^{N}-g \mathbf{H}_{I}(\rho)\right) \mathcal{U}^{-1}(g \rho)=I \oslash \mathbf{H}_{0}^{N}+g V_{\epsilon f f}
$$

In subsection 3.2, we give the definition of the Hamiltonian $\mathbf{H}_{\rho}^{N}$. Using the unitary equivalence (1.6), as in the Pauli-Fierz model, we get (1.4). From (1.4) we get some inequalities as that of the Pauli-Fierz model.

In section 4 , we give some remarks about the both of the Pauli-Fierz and the Nelson models; the dipole approximation, essentially self-adjointness of $\mathbf{H}_{\rho}^{P}$ and an effective potential appearing in a scaling limit of the Hamiltonian $\mathbf{H}_{\rho}^{N}$. 


\section{PART I: THE PAULI-FIERZ MODEL OF QED 2.1 GAUGE TRANSFORMATION}

Let $\mathcal{H}$ be a Hilbert space. We denote the inner product and the associated norm by $(*, \cdot)_{\mathcal{H}}$ and $\|\cdot\|_{\mathcal{H}}$ respectively. The inner product is linear in $\cdot$ and antilinear in $*$. The domain of an operator $A$ is denoted by $D(A)$. For $f \in L^{2}\left(\mathbb{R}^{d}\right), \hat{f}$ (resp. $f$ ) is the Fourier transformation (resp. the inverse Fourier transformation) of $f$ and $\bar{f}$ the complex conjugate of $f$.

We define the Boson Fock space $\mathcal{F}(\mathcal{W})$ over $\mathcal{W}=\underbrace{L^{2}\left(\mathbb{R}^{d}\right) \oplus \ldots \oplus L^{2}\left(\mathbb{F}^{d}\right)}_{d-1}$ by $([10,11,12])$

$$
\begin{aligned}
\mathcal{F}(\mathcal{W}) & \equiv \bigoplus_{n=0}^{\infty} \otimes_{s}^{n} \mathcal{W} \\
& \equiv \bigoplus_{n=0}^{\infty} \mathcal{F}_{n}(\mathcal{W})
\end{aligned}
$$

where $\otimes_{s}^{n}$ denotes the $n$-fold symmetric tensor product with $\otimes_{s}^{0} \mathcal{W}=\mathbb{C}$. Let

$$
\mathcal{F}^{N}(\mathcal{W})=\bigoplus_{n=0}^{N} \mathcal{F}^{n}(\mathcal{W}) \bigoplus_{n \geq N+1}\{0\}
$$

Then finite particle subspace is defined by

$$
\mathcal{F}^{\infty}(\mathcal{W})=\bigcup_{N=0}^{\infty} \mathcal{F}^{N}(\mathcal{W})
$$

We call $\Omega=\{1,0,0, \ldots\}$ the vacuum vector. We denote the annihilation operator and the creation operator by $a^{\dagger}(f)$ and $a(f), f \in \mathcal{W}$, respectively, where $a^{\sharp}(\cdot)$ ( $a^{\sharp}$ denotes either $a$ or $\left.a^{\sharp}\right)$ is linear in $\cdot$ It is known that $a^{\sharp}(f)$ is well defined on $\mathcal{F}^{\infty}(\mathcal{W})$ and leave it invariant. Also the following commutation relations (CCR) are satisfied;

$$
\begin{aligned}
{\left[a(f), a^{\dagger}(g)\right] \Phi=} & (\bar{f}, g)_{\mathcal{W}} \Phi \\
{\left[a^{\dagger}(f), a^{\dagger}(g)\right] \Phi=} & {[a(f), a(g)] \Phi=0, } \\
& \Phi \in \mathcal{F}^{\infty}(\mathcal{W}), f, g \in \mathcal{W}
\end{aligned}
$$


where $[A, B]=A B-B A$. Furthermore, the following adjoint relation holds

$$
(a(f) \Phi, \Psi)_{\mathcal{F}(\mathcal{W})}=\left(\Phi, a^{\dagger}(\bar{f}) \Psi\right)_{\mathcal{F}(\mathcal{W})}, \quad \Phi, \Psi \in \mathcal{F}^{\infty}(\mathcal{W}), f, g \in \mathcal{W}
$$

It is the fundamental fact that

$$
\mathcal{F}(\mathcal{W})=\left\{a^{\dagger}\left(f_{1}\right) \ldots a^{\dagger}\left(f_{n}\right) \Omega, \Omega \mid f_{j} \in \mathcal{W}, j=1, \ldots, n, n \geq 1\right\}^{-}
$$

where \{\}$^{-}$denotes the closure of \{\} in $\mathcal{F}(\mathcal{W})$. For any nonnegative self-adjoint operator $h$ in $\mathcal{W}$, there exists a strongly continuous one parameter semigroup $\left\{\Gamma\left(e^{-t h}\right)\right\}_{t \geq 0}$ on $\mathcal{F}(\mathcal{W})$ such that

$$
\begin{aligned}
\Gamma\left(e^{-t h}\right) a^{\dagger}\left(f_{1}\right) \ldots a^{\dagger}\left(f_{n}\right) \Omega & =a^{\dagger}\left(e^{-t h} f_{1}\right) \ldots a^{\dagger}\left(e^{-t h} f_{n}\right) \Omega \\
\Gamma\left(e^{-t h}\right) \Omega & =0, f_{j} \in \mathcal{W}, j=1, \ldots, n
\end{aligned}
$$

By Stone's theorem ([18,Theorem VIII.8]), there exists a nonnegative self-adjoint operator $d \Gamma(h)$ such that

$$
e^{-t d \Gamma(h)}=\Gamma\left(e^{-t h}\right)
$$

We call $d \Gamma(h)$ the "second quantization of $h$ ". We define a multiplication operator $\omega: L^{2}\left(\mathbb{R}^{d}\right) \rightarrow L^{2}\left(\mathbb{R}^{d}\right)$ as follows:

$$
(\omega \hat{f})(k)=|k| \hat{f}(k)
$$

Put $\tilde{\omega}=\underbrace{\omega \oplus \ldots \oplus \omega}_{d-1}$. The free Hamiltonian $\mathbf{H}_{0}^{P}$ is defined by

$$
\mathbf{H}_{0}^{P}=d \Gamma(\tilde{\omega})
$$

Set $\mathbf{N}_{P}=d \Gamma(I)$, where $I$ denotes the identity operator on $\mathcal{W}$. It is called the "number operator". The following inequality is fundamental;

$$
\left\|a^{\sharp}(f) \Phi\right\|_{\mathcal{F}(\mathcal{W})} \leq\|f\|_{\mathcal{W}}\left\|\left(\mathbf{N}_{P}+1\right)^{\frac{1}{2}} \Phi\right\|_{\mathcal{F}(\mathcal{W})}, \quad \Phi \in D\left(\mathbf{N}_{P}^{\frac{1}{2}}\right), f \in \mathcal{W} .
$$


Let $e^{r}: \mathbb{R}^{d} \rightarrow \mathbb{R}^{d}(r=1, \ldots, d-1)$ be a measurable function such that

$$
k \cdot e^{r}(k)=0, \quad e^{r}(k) \cdot e^{s}(k)=\delta_{r s}, \quad \text { a.e.k } \in \mathbb{R}^{d}
$$

where $\delta_{r s}$ is Kroneker's delta. The vector-valued function $\epsilon^{r}$ serves as a polarization vector of photons. We denote the $\mu$-th component of $e^{r}$ by $e_{\mu}^{r}(\mu=1, \ldots, d)$. Let

$$
\begin{aligned}
d_{\mu \nu}(k) & \equiv \sum_{r=1}^{d-1} e_{\mu}^{r}(k) e_{\nu}^{r}(k) \\
& =\delta_{\mu \nu}-\frac{k_{\mu} k_{\nu}}{|k|^{2}}
\end{aligned}
$$

Put

$$
\mathbb{D}_{n}=\left\{\left.f\left|\int_{\mathbb{R}^{d}}\right| k\right|^{n}|f(k)|^{2} d k<\infty\right\}
$$

Then, for each $x \in \mathbb{R}^{d}$, the time-zero radiation field and the conjugate momentum are defined by $([9,11])$

$$
\begin{aligned}
& A_{\mu}(x, \hat{f})=\frac{1}{\sqrt{2}}\left\{a^{\dagger}\left(\oplus_{r=1}^{d-1} e_{\mu}^{r} \frac{\hat{f}}{\sqrt{\omega}} e^{-i \cdot x}\right)+a\left(\oplus_{r=1}^{d-1} e_{\mu}^{r} \frac{\tilde{\hat{f}}}{\sqrt{\omega}} e^{i \cdot x}\right)\right\}, \hat{f} \in \mathbb{D}_{-1}, \\
& \Pi_{\mu}(x, \hat{f})=\frac{i}{\sqrt{2}} \sum_{r=1}^{d-1}\left\{a^{\dagger}\left(\oplus_{r=1}^{d-1} e_{\mu}^{r} \hat{f} \sqrt{\omega} \epsilon^{-i \cdot x}\right)-a\left(\oplus_{r=1}^{d-1} e_{\mu}^{r} \tilde{\hat{f}} \sqrt{\omega} e^{i \cdot x}\right)\right\}, \hat{f} \in \mathbb{L}_{1},
\end{aligned}
$$

where $\tilde{g}(k)=g(-k)$. When $f$ is a real valued function, by (2.1) and the Nelson analytic vector theorem $\left(\left[19\right.\right.$, Theorem X.39]), for each $x \in \mathbb{R}^{d}, A_{\mu}(x, \hat{f})$ and $\Pi_{\mu}(x, \hat{f})$ are essentially self-adjoint on $\mathcal{F}^{\infty}(\mathcal{W})$. We denote the closures by the same symbols. Using CCR, we have the following commutation relations on $\mathcal{F}^{\infty}(\mathcal{W})$;

$$
\begin{aligned}
{\left[A_{\mu}(x, \hat{f}), \Pi_{\nu}(x, \hat{g})\right]=} & i\left(d_{\mu \nu} \hat{\bar{f}}, \hat{g}\right)_{L^{2}\left(\mathbb{R}^{d}\right)}, \hat{f} \in \mathbb{D}_{-1} \cap \mathbb{D}_{0}, \hat{g} \in \mathbb{D}_{1} \cap \mathbb{D}_{0}, \\
{\left[A_{\mu}(x, \hat{f}), A_{\nu}(x, \hat{g})\right]=} & 0, \hat{f}, \hat{g} \in \mathbb{D}_{-1} \\
{\left[\Pi_{\mu}(x, \hat{f}), \Pi_{\nu}(x, \hat{g})\right]=} & 0, \hat{f}, \hat{g} \in \mathbb{D}_{1} \\
& \mu, \nu=1, \ldots, d
\end{aligned}
$$


We set

$$
\mathcal{M} \equiv L^{2}\left(\mathbb{R}^{d}\right) \otimes \mathcal{F}(\mathcal{W}) \cong \int_{\mathbb{R}^{d}}^{\oplus} \mathcal{F}(\mathcal{W}) d x
$$

The Hilbert space $\mathcal{M}$ denotes the set of the state vectors of a quantum system of a nonrelativistic charged particle interacting with a quantized radiation field. Put

$$
\begin{aligned}
& \int_{\mathbb{R}^{d}}^{\oplus} A_{\mu}(x, \hat{f}) d x \equiv A_{\mu}(\hat{f}), \\
& \int_{\mathbb{R}^{d}}^{\oplus} \Pi_{\mu}(x, \hat{f}) d x \equiv \Pi_{\mu}(\hat{f}) .
\end{aligned}
$$

Let $D_{\mu}$ be the generalized $L^{2}$-derivative in the $\mu$-th direction. By (2.1), it can be easily seen that the following commutation relations hold on $C^{1}\left(\mathbb{R}^{d} ; D\left(\mathbf{N}_{P}^{\frac{1}{2}}\right)\right)$ (the set of $D\left(\mathbf{N}_{P}^{\frac{1}{2}}\right)$-valued strongly continuously-differentiable functions);

$$
\begin{aligned}
& {\left[D_{\mu} \otimes I, A_{\nu}(\hat{f})\right]=-\Pi_{\nu}\left(k_{\mu} \frac{\hat{f}}{\omega}\right), \hat{f} \in \mathbb{D}_{-1}, k_{\mu} \hat{f} \in \mathbb{D}_{-1}} \\
& {\left[D_{\mu} \otimes I, \Pi_{\nu}(\hat{f})\right]=A_{\nu}\left(k_{\mu} \omega \hat{f}\right), \hat{f} \in \mathbb{D}_{1}, k_{\mu} \hat{f} \in \mathbb{D}_{1}}
\end{aligned}
$$

Let

$$
\begin{aligned}
\tilde{\Pi}_{\mu}(x, \hat{f}) & \equiv \Pi_{\mu}\left(x, \frac{\hat{f}}{k_{\mu} \omega}\right) \\
& =\frac{i}{\sqrt{2}}\left\{a^{\dagger}\left(\oplus_{r=1}^{d-1} e_{\mu}^{r} \frac{\hat{f}_{\mu}}{\sqrt{\omega}} e^{-i \cdot x}\right)-a\left(\oplus_{r=1}^{d-1} e_{\mu}^{r} \frac{\tilde{\hat{f}}_{\mu}}{\sqrt{\omega}} e^{i \cdot x}\right)\right\}, \hat{f}_{\mu} \in \mathbb{D}_{-1}
\end{aligned}
$$

where

$$
\hat{f}_{\mu}(k)=\frac{\hat{f}(k)}{k_{\mu}}
$$

Put

$$
\int_{\mathbb{R}^{d}}^{\oplus} \tilde{\Pi}_{\mu}(x, \hat{f}) d x \equiv \tilde{\Pi}_{\mu}(\hat{f})
$$

and

$$
\mathbb{D}_{-1, \mu}=\left\{f \mid \check{f} \text { is real valued and } f_{\mu} \in \mathbb{D}_{-1}\right\} \text {. }
$$


For $\hat{f} \in \mathbb{D}_{-1, \mu}$, we define unitary operators $\mathcal{U}_{\mu}(f), \mu=1, \ldots, d$ on $\mathcal{M}$ by

$$
\mathcal{U}_{\mu}(f)=\exp \left(i \tilde{\Pi}_{\mu}(\hat{f})\right)
$$

Set $\mathcal{D}=C_{0}^{\infty}\left(\mathbb{R}^{d}\right) \hat{\otimes} \mathcal{F}^{\infty}(\mathcal{W})(\hat{\otimes}$ is the algebraic tensor product $)$

Lemma 2.1 Let $\hat{f} \in \mathbb{D}_{-1, \mu} \cap \mathbb{D}_{-1}$. Then

$$
\mathcal{U}_{\mu}(f) \mathcal{D} \subset D\left(D_{\mu} \otimes I\right)
$$

Furthermore, the following commutation relation holds on $\mathcal{D}$;

$$
\left[D_{\mu} \otimes I, \mathcal{U}_{\mu}(f)\right]=i A_{\mu}(\hat{f}) \mathcal{U}_{\mu}(f)
$$

Proof: Since $\mathcal{D}$ is the set of analytic vectors of $\tilde{\Pi}_{\mu}(\hat{f})$, we have on $\mathcal{D}$

$$
\mathcal{U}_{\mu}(f)=s-\lim _{N \rightarrow \infty} \sum_{n=0}^{N} \frac{\left(i \tilde{\Pi}_{\mu}(\hat{f})\right)^{n}}{n !} .
$$

On the other hand, by $(2.3)$, on $\mathcal{D}$, we have

$$
\begin{aligned}
& \left(D_{\mu} \otimes I\right) \sum_{n=0}^{N} \frac{\left(i \tilde{\Pi}_{\mu}(\hat{f})\right)^{n}}{n !} \\
& =\sum_{n=1}^{N} \sum_{j=0}^{n-1} \frac{\left(i \tilde{\Pi}_{\mu}(\hat{f})\right)^{j}\left[D_{\mu} \otimes I, i \tilde{\Pi}_{\mu}(\hat{f})\right]\left(i \tilde{\Pi}_{\mu}(\hat{f})\right)^{n-j-1}}{n !}+\sum_{n=0}^{N} \frac{\left(i \tilde{\Pi}_{\mu}(\hat{f})\right)^{n}}{n !}\left(D_{\mu} \otimes I\right) .
\end{aligned}
$$

Note that on $\mathcal{D}$

$$
\begin{aligned}
{\left[\left[D_{\mu} \otimes I, i \tilde{\Pi}_{\mu}(\hat{f})\right], i \tilde{\Pi}_{\mu}(\hat{f})\right] } & =\left[i A_{\mu}(\hat{f}), i \tilde{\Pi}_{\mu}(\hat{f})\right] \\
& =-i\left(d_{\mu \mu} \frac{\hat{f}_{\mu}}{\sqrt{\omega}}, \frac{\hat{f}}{\sqrt{\omega}}\right)_{L^{2}\left(\mathbb{R}^{d}\right)} \\
& =0
\end{aligned}
$$

Then the right hand side(r.h.s.) of (2.4) is as follows:

$$
i A_{\mu}(\hat{f}) \sum_{n=0}^{N-1} \frac{\left(i \tilde{\Pi}_{\mu}(\hat{f})\right)^{n}}{n !}+\sum_{n=0}^{N} \frac{\left(i \tilde{\Pi}_{\mu}(\hat{f})\right)^{n}}{n !}\left(D_{\mu} \odot I\right)
$$


By (2.1), it follows that (2.5) strongly converges to

$$
i A_{\mu}(\hat{f}) e^{i \widetilde{\Pi}_{\mu}(\hat{f})}+e^{i \widetilde{\Pi}_{\mu}(\hat{f})} D_{\mu} \oslash I
$$

on $\mathcal{D}$ as $n \rightarrow \infty$. Since $D_{\mu} \otimes I$ is closed, the lemma follows.

From Lemma 2.1 it follows that on $\mathcal{U}_{\mu}(f) \mathcal{D}$

$$
\mathcal{U}_{\mu}(f)\left(-i D_{\mu} \otimes I\right) \mathcal{U}_{\mu}(f)^{-1}=-i D_{\mu} \otimes I-A_{\mu}(\hat{f})
$$

Since $-i D_{\mu} \otimes I$ leaves $\mathcal{D}$ invariant, we see that on $\mathcal{U}_{\mu}(f) \mathcal{D}$

$$
\mathcal{U}_{\mu}(f)\left(-\Delta_{\mu} \otimes I\right) \mathcal{U}_{\mu}(f)^{-1}=\left(-i D_{\mu} \otimes I-A_{\mu}(\hat{f})\right)^{2}
$$

where $-\Delta_{\mu}=-D_{\mu} D_{\mu}$. Since $\mathcal{D}$ is the core of $-\Delta_{\mu} \otimes I$, it follows that the r.h.s.of (2.6) is essentially self-adjoint on $\mathcal{U}_{\mu}(f) \mathcal{D}$. Hence

$$
\mathbf{H}_{\mu}(f) \equiv \overline{\left.\frac{1}{2}\left(-i D_{\mu} \otimes I-A_{\mu}(\hat{f})\right)^{2}\right|_{\mathcal{U}_{\mu}(f) \mathcal{D}}}
$$

is self-adjoint. Obviously $\mathbf{H}_{\mu}(f)$ is nonnegative. Thus we have obtained the following theorem.

Theorem 2.2 Let $\hat{f} \in \mathbb{D}_{-1, \mu} \cap \mathbb{D}_{-1}$. Then $\mathcal{U}_{\mu}(f)^{-1}$ maps $D\left(\mathbf{H}_{\mu}(f)\right)$ onto $D\left(-\frac{1}{2} \Delta_{\mu} \otimes I\right)$ with

$$
\mathcal{U}_{\mu}(f)\left(-\frac{1}{2} \Delta_{\mu} \otimes I\right) \mathcal{U}_{\mu}(f)^{-1}=\mathbf{H}_{\mu}(f), \quad \mu=1, \ldots, d
$$

\subsection{DIAMAGNETIC INEQUALITY}

In this subsection, we define a self-adjoint Hamiltonian for the Pauli-Fierz model. Also we derive diamagnetic inequalities. 
For $\hat{\rho} \in \cap_{\mu} \mathbb{D}_{-1, \mu} \cap \mathbb{D}_{-1}$, the Hamiltonian $\mathbf{H}_{\rho}^{P}$ for the Pauli-Fierz model with an ultraviolet cut-off function $\rho$ is defined by a self-adjoint operator acting in $\mathcal{M}$ as follows:

$$
\mathbf{H}_{\rho}^{P}=\mathbf{H}_{1}(\rho) \dot{+} \ldots \dot{+} \mathbf{H}_{d}(\rho) \dot{+} I \ominus \mathbf{H}_{0}^{P}
$$

where $\dot{+}$ means a form sum.

Theorem 2.3 (Diamagnetic inequality) Let $V \in L^{\infty}\left(\mathbb{R}^{d}\right)$ and $\hat{\rho} \in \cap_{\mu} \mathbb{D}_{-1, \mu} \cap \mathbb{D}_{-1}$. Then

$$
\left|\left(F, e^{-t\left(\mathbf{H}_{\rho}^{P}+V \otimes I\right)} G\right)_{\mathcal{M}}\right| \leq\left(\|F\|_{\mathcal{F}(\mathcal{W})}, e^{-t\left(-\frac{1}{2} \Delta+V\right)}\|G\|_{\mathcal{F}(\mathcal{W})}\right)_{L^{2}\left(\mathbb{R}^{d}\right)} .
$$

Proof: By the strong Trotter product formula([20]) and Theorem 2.2, we see that

$$
\begin{aligned}
& \left(F, e^{-t\left(\mathbf{H}_{\rho}^{P}+V \otimes I\right)} G\right)_{\mathcal{M}} \\
& =\lim _{n \rightarrow \infty}\left(F,\left(\mathcal{U}_{1}(\rho) e^{-\frac{t}{n}\left(\frac{-1}{2} \Delta_{1} \otimes I\right)} \mathcal{U}_{1}(\rho)^{-1} \ldots .\right.\right. \\
& \left.\left.\ldots \ldots \mathcal{U}_{d}(\rho) e^{-\frac{t}{n}\left(\frac{-1}{2} \Delta_{d} \otimes I\right)} \mathcal{U}_{d}(\rho)^{-1} e^{-\frac{t}{n} I \otimes \mathbf{H}_{0}^{P}} e^{-\frac{t}{n} V \otimes I}\right)^{n} G\right)_{\mathcal{M}} .
\end{aligned}
$$

Note that for $H \in \mathcal{M}$ and $x \in \mathbb{R}^{d}$,

$$
\begin{aligned}
\left\|\left(\mathcal{U}_{\mu}(\rho) H\right)(x)\right\|_{\mathcal{F}(\mathcal{W})} & =\left\|\mathcal{U}_{\mu}(x, \rho) H(x)\right\|_{\mathcal{F}(\mathcal{W})} \\
& =\|H(x)\|_{\mathcal{F}(\mathcal{W})}
\end{aligned}
$$

where

$$
\mathcal{U}_{\mu}(x, \rho)=\exp \left(i \tilde{\Pi}_{\mu}(x, \hat{\rho})\right)
$$

It is well known that for $H \in \mathcal{M}$ and $x \in \mathbb{R}^{d}$,

$$
\left(e^{-t\left(-\frac{1}{2} \Delta_{\mu} \otimes I\right)} H\right)(x)=\int_{\mathbb{R}} \frac{1}{\sqrt{2 \pi t}} e^{\frac{-\left|x_{\mu}-y_{\mu}\right|^{2}}{2 t}} H(y) d y, \text { a.e. } x \in \mathbb{R}^{d},
$$

where the integral is in the Bochner sense. Hence we have

$$
\left\|\left(e^{-t\left(-\frac{1}{2} \Delta_{\mu} \otimes I\right)} H\right)(x)\right\|_{\mathcal{F}(\mathcal{W})} \leq\left(e^{-t\left(-\frac{1}{2} \Delta_{\mu}\right)}\|H(\cdot)\|_{\mathcal{F}(\mathcal{W})}\right)(x), \quad \text { a.e..x } \in \mathbb{R}^{d}
$$


Combining (2.7) and (2.8), and noting that $\exp \left(-t \Delta_{\mu}\right)$ is positivity preserving, we have for all $F, G \in \mathcal{M}$,

$$
\begin{aligned}
& \left|\left(F, e^{-t\left(\mathbf{H}_{\rho}^{P}+V \otimes I\right)} G\right)_{\mathcal{M}}\right| \\
& \leq \lim _{n \rightarrow \infty}\left(\|F\|_{\mathcal{F}(\mathcal{W})},\left(e^{-\frac{t}{n}\left(-\frac{1}{2} \Delta\right)} e^{-\frac{t}{n} V}\right)^{n}\|G\|_{\mathcal{F}(\mathcal{W})}\right)_{L^{2}\left(\mathbb{R}^{d}\right)} \\
& =\left(\|F\|_{\mathcal{F}(\mathcal{W})}, e^{-t\left(-\frac{1}{2} \Delta+V\right)}\|G\|_{\mathcal{F}(\mathcal{W})}\right)_{L^{2}\left(\mathbb{R}^{d}\right)}
\end{aligned}
$$

Lemma 2.4 Let $|V|$ be a multiplication operator which is $-\frac{1}{2} \Delta$-form bounded with relative bound $\epsilon$. Then for $\hat{\rho} \in \cap_{\mu} \mathbb{D}_{-1, \mu} \cap \mathbb{D}_{-1},|V|$ is $\mathbf{H}_{\rho}^{P}$-form bounded with the relative bound at most $\epsilon$.

Proof: It is similar to the proof of [12,Lemma 4.11].

For the multiplication operator $V$, let us introduce a class of potentials as follows:

Definition 2.5 A potential $V \in P$ if and only if $V_{+} \in L_{l o c}^{1}\left(\mathbb{R}^{d}\right)$ and $V_{-}$is $-\frac{1}{2} \Delta$-form bounded with relative bound $<1$. Here

$$
\left\{\begin{array}{l}
V_{+}=\max \{0, V\}, \\
V_{-}=\max \{0,-V\} .
\end{array}\right.
$$

For $V \in P$, by Lemma 2.4 , one can define a self-adjoint operator

$$
\mathbf{H}_{\rho}^{P} \dot{+} V_{+}-V_{-}
$$

Theorem 2.6 Let $V \in P$ and $\hat{\rho} \in \cap_{\mu} \mathbb{D}_{-1, \mu} \cap \mathbb{D}_{-1}$. Then Theorem 2.3 holds with $\mathbf{H}_{\rho}^{P}+V$ replaced by $\mathbf{H}_{\rho}^{P} \dot{+} V_{+} \dot{-} V_{-}$.

Proof: It is similar to the proof of [12, Theorem 4.13].

Let $\sigma(A)$ denote the spectrum of $A$. We can obtain the following corollaries in the same way as in [12]. 
Corollary 2.7 Let $V \in P$ and $\hat{\rho} \in \cap_{\mu} \mathbb{D}_{-1, \mu} \cap \mathbb{D}_{-1}$. Then

$$
\inf \sigma\left(-\frac{1}{2} \Delta \dot{+} V_{+} \dot{-} V_{-}\right) \leq \inf \sigma\left(\mathbf{H}_{\rho}^{P} \dot{+} V_{+}-V_{-}\right)
$$

Corollary 2.8 (Abstract Kato's inequality) Let $V \in P$ and $\hat{\rho} \in \cap_{, \mu} \mathbb{L}_{-1, \mu} \cap \mathbb{D}_{-1}$. Suppose. $\psi \in D\left(\left(-\frac{1}{2} \Delta \dot{+} V_{+} \dot{-} V_{-}\right)^{\frac{1}{2}}\right), \psi \geq 0$ and $G \in D\left(\mathbf{H}_{\rho}^{P} \dot{+} V_{+} \dot{-} V_{-}\right)$.

Then $\|G(\cdot)\|_{\mathcal{F}(\mathcal{W})} \in D\left(\left(-\frac{1}{2} \Delta \dot{+} V_{+}-V_{-}\right)^{\frac{1}{2}}\right)$ and the following inequality holds;

$$
\begin{aligned}
& \Re\left((\operatorname{sign} G) \psi,\left(\mathbf{H}_{\rho}^{P} \dot{+} V_{+}-V_{-}\right) G\right)_{\mathcal{M}} \\
& \geq\left(\left(-\frac{1}{2} \Delta \dot{+} V_{+} \dot{-} V_{-}\right)^{\frac{1}{2}} \psi,\left(-\frac{1}{2} \Delta \dot{+} V_{+} \dot{-} V_{-}\right)^{\frac{1}{2}}\|G\|_{\mathcal{F}(W)}\right)_{L^{2}\left(\mathbb{R}^{d}\right)}
\end{aligned}
$$

where

$$
(\operatorname{sign} G)(x)= \begin{cases}\frac{G(x)}{\|G(x)\|_{\mathcal{F}(\mathcal{W})},}, & \|G(x)\|_{\mathcal{F}(\mathcal{W})} \neq 0 \\ 0, & \|G(x)\|_{\mathcal{F}(\mathcal{W})}=0\end{cases}
$$

\section{PART II: THE NELSON MODEL}

\subsection{BOGOLIUBOV TRANSFORMATION}

Let $\mathcal{F}=\mathcal{F}\left(L^{2}\left(\mathbb{R}^{d}\right)\right)$ denote the Boson Fock space over $L^{2}\left(\mathbb{R}^{d}\right)$. As in PART I, the finite particle subspace in $\mathcal{F}$ is denoted by $\mathcal{F}^{\infty}$. We define a multiplication operator $\omega_{\mu}: L^{2}\left(\mathbb{E}^{d}\right) \rightarrow$ $L^{2}\left(\mathbb{R}^{d}\right)$ by

$$
\left(\omega_{\mu} \hat{f}\right)(k)=\sqrt{k^{2}+\mu^{2}} \hat{f}(k)
$$

where $\mu>0$ is a constant which denotes the mass of a meson. We define the free Hamiltonian $\mathbf{H}_{0}^{N}$ in $\mathcal{F}$ by the second quantization of $\omega_{\mu}$ :

$$
\mathbf{H}_{0}^{N}=d \Gamma\left(\omega_{\mu}\right)
$$


We denote the number operator by $\mathbf{N}_{N}$. Let

$$
\mathbb{D}_{n}^{\mu}=\left\{\left.f\left|\int_{\mathbb{R}^{d}} \omega_{\mu}^{n}(k)\right| f(k)\right|^{2} d k<\infty\right\} .
$$

Let $a(f)$ and $a^{\dagger}(f)$ be the annihilation operator and the creation operator on $\mathcal{F}$ respectively, (we often use the same notations as that of the Pauli-Fierz model in PART I, if no confusions arising) which satisfy the following canonical commutation relations on $\mathcal{F}^{\infty}$;

$$
\begin{aligned}
{\left[a(f), a^{\dagger}(g)\right] } & =(\bar{f}, g)_{L^{2}\left(\mathbb{R}^{d}\right)}, \\
{\left[a^{\dagger}(f), a^{\dagger}(g)\right] } & =[a(f), a(g)]=0 .
\end{aligned}
$$

It is well known that

$$
\begin{aligned}
\left\|a^{\sharp}(f) \Phi\right\|_{\mathcal{F}} & \leq\left\|f / \sqrt{\omega_{\mu}}\right\|_{L^{2}\left(\mathbb{R}^{d}\right)}\left\|\left(\mathbf{H}_{0}^{N}\right)^{\frac{1}{2}} \Phi\right\|_{\mathcal{F}}+\|f\|_{L^{2}\left(\mathbb{R}^{d}\right)}\|\Phi\|_{\mathcal{F}}, \Phi \in D\left(\left(\mathbf{H}_{0}^{N}\right)^{\frac{1}{2}}\right), \\
\left\|a^{\sharp}(f) \Psi\right\|_{\mathcal{F}} & \leq\|f\|_{L^{2}\left(\mathbb{R}^{d}\right)}\left\|\left(\mathbf{N}_{N}+I\right)^{\frac{1}{2}} \Psi\right\|_{\mathcal{F}}, \Psi \in D\left(\mathbf{N}_{N}^{\frac{1}{2}}\right) .
\end{aligned}
$$

Furthermore, for $f \in \mathbb{D}_{-1}^{\mu} \cap \mathbb{D}_{0}^{\mu} \cap \mathbb{D}_{1}^{\mu} \cap \mathbb{D}_{2}^{\mu}, a^{\sharp}(f)$ maps $D\left(\left(\mathbf{H}_{0}^{N}\right)^{\frac{3}{2}}\right)$ into $D\left(\mathbf{H}_{0}^{N}\right)$ with

$$
\begin{aligned}
{\left[\mathbf{H}_{0}^{N}, a^{\dagger}(f)\right] \Phi=} & a^{\dagger}\left(\omega_{\mu} f\right) \Phi \\
{\left[\mathbf{H}_{0}^{N}, a(f)\right] \Phi=} & -a\left(\omega_{\mu} f\right) \Phi \\
& \Phi \in D\left(\left(\mathbf{H}_{0}^{N}\right)^{\frac{3}{2}}\right)
\end{aligned}
$$

Also for $f \in \cap_{n=-1}^{\infty} \mathbb{D}_{n}^{\mu}, a^{\sharp}(f)$ leaves $C^{\infty}\left(\mathbf{H}_{0}^{N}\right)=\cap_{n=1}^{\infty} D\left(\left(\mathbf{H}_{0}^{N}\right)^{n}\right)$ invariant. The time-zero field and the conjugate momentum are defined by

$$
\begin{aligned}
& A(x, \hat{f})=\frac{1}{\sqrt{2}}\left\{a^{\dagger}\left(\frac{\hat{f}}{\sqrt{\omega_{\mu}}} e^{-i \cdot x}\right)+a\left(\frac{\tilde{\hat{f}}}{\sqrt{\omega_{\mu}}} e^{i \cdot x}\right)\right\}, \hat{f} \in \mathbb{D}_{-1}^{\mu}, \\
& \Pi(x, \hat{f})=\frac{i}{\sqrt{2}}\left\{a^{\dagger}\left(\hat{f} \sqrt{\omega_{\mu}} e^{-i \cdot x}\right)-a\left(\tilde{\hat{f}} \sqrt{\omega_{\mu}} e^{i \cdot x}\right)\right\}, \hat{f} \in \mathbb{D}_{1}^{\mu}
\end{aligned}
$$

The time-zero field and the conjugate momentum satisfy the following commutation relations on $\mathcal{F}^{\infty}$;

$$
[A(x, \hat{f}), \Pi(x, \hat{g})]=i(\hat{\bar{f}}, \hat{g})_{L^{2}\left(\mathbb{R}^{d}\right)}, \hat{f} \in \mathbb{D}_{-1}^{\mu} \cap \mathbb{D}_{0}^{\mu}, \hat{g} \in \mathbb{D}_{0}^{\mu} \cap \mathbb{D}_{1}^{\mu}
$$




$$
\begin{aligned}
& {[A(x, \hat{f}), A(x, \hat{g})]=0, \hat{f}, \hat{g} \in \mathbb{D}_{-1}^{\mu},} \\
& {[\Pi(x, \hat{f}), \Pi(x, \hat{g})]=0, \hat{f}, \hat{g} \in \mathbb{D}_{1}^{\mu} .}
\end{aligned}
$$

From (3.3) it follows that on $D\left(\left(\mathbf{H}_{0}^{N}\right)^{\frac{3}{2}}\right)$

$$
\begin{aligned}
{\left[\mathbf{H}_{0}^{N}, A(x, \hat{f})\right] } & =-i \Pi(x, \hat{f}), \hat{f} \in \mathbb{D}_{-2}^{\mu} \cap \mathbb{D}_{-1}^{\mu} \cap \mathbb{D}_{0}^{\mu} \cap \mathbb{D}_{1}^{\mu}, \\
{\left[\mathbf{H}_{0}, \Pi(x, \hat{f})\right] } & =i A\left(x, \omega_{\mu}^{2} \hat{f}\right), \hat{f} \in \mathbb{D}_{0}^{\mu} \cap \mathbb{D}_{1}^{\mu} \cap \mathbb{D}_{2}^{\mu} \cap \mathbb{D}_{3}^{\mu} .
\end{aligned}
$$

For each $x_{j} \in \mathbb{R}^{d}, j=1, \ldots, N$ and a real valued function $f$ with $\hat{f} \in \mathbb{D}_{-1}^{\mu}\left(\right.$ resp. $\left.\hat{f} \in \mathbb{W}_{1}^{\prime \prime}\right)$, $\sum_{j=1}^{N} A\left(x_{j}, \hat{f}\right)$ (resp. $\left.\sum_{j=1}^{N} \Pi\left(x_{j}, \hat{f}\right)\right)$ is essentially self-adjoint on $\mathcal{F}^{\infty}$. We denote the selfadjoint extensions by the same symbols. For a real valued function $f$ with $\hat{f} \in \mathbb{D}_{-3}^{\mu}$, put

$$
\begin{aligned}
\tilde{\Pi}(x, \hat{f}) & \equiv \Pi\left(x, \frac{\hat{f}}{\omega_{\mu}^{2}}\right), \\
& =\frac{i}{\sqrt{2}}\left\{a^{\dagger}\left(\frac{\hat{f}}{\omega_{\mu}^{2}} \sqrt{\omega_{\mu}} e^{-i \cdot x}\right)-a\left(\frac{\hat{f}}{\omega_{\mu}^{2}} \sqrt{\omega_{\mu}} e^{i \cdot x}\right)\right\} .
\end{aligned}
$$

Then we define a unitary operator $\mathcal{U}\left(x_{1}, \ldots, x_{N}, f\right)$ on $\mathcal{F}$ by

$$
\mathcal{U}\left(x_{1}, \ldots, x_{N}, f\right)=\exp \left(i \sum_{j=1}^{N} \tilde{\Pi}\left(x_{j}, \hat{f}\right)\right) .
$$

Lemma 3.1 Let $f$ be a real valued function with $\hat{f} \in \mathbb{D}_{-4}^{\mu} \cap \mathbb{D}_{-3}^{\mu} \cap \mathbb{D}_{-2}^{\mu} \cap \mathbb{D}_{-1}^{\mu}$. Then $\mathcal{U}\left(x_{1}, \ldots, x_{N}, f\right)$ maps $D\left(\mathbf{H}_{0}^{N}\right)$ onto itself with

$$
\mathcal{U}^{-1}\left(x_{1}, \ldots, x_{N}, f\right) \mathbf{H}_{0}^{N} \mathcal{U}\left(x_{1}, \ldots, x_{N}, f\right)=\mathbf{H}_{0}^{N}-\sum_{j=1}^{N} A\left(x_{j}, \hat{f}\right)-V_{e f f}\left(x_{1}, \ldots, x_{N}, f\right),
$$

where

$$
V_{e f f}\left(x_{1}, \ldots, x_{N}, f\right)=-\frac{1}{2}\left\|\frac{\hat{f}}{\omega_{\mu}} \sum_{j=1}^{N} e^{-i \cdot x_{j}}\right\|_{L^{2}\left(\mathbb{R}^{d}\right)}^{2} .
$$

Proof: For simplicity, put $\sum_{j=1}^{N} \tilde{\Pi}\left(x_{j}, \hat{f}\right)=\tilde{\Pi}(\hat{f})$. Since $C^{\infty}\left(\mathbf{H}_{0}^{N}\right) \cap \mathcal{F}^{\infty}$ is the set of the analytic vectors for $\tilde{\Pi}(\hat{f})$, for $\Phi, \Psi \in C^{\infty}\left(\mathbf{H}_{0}^{N}\right) \cap \mathcal{F}^{\infty}$ and a real valued function $f$ with 
$\hat{f} \in \cap_{n=-4}^{\infty} \mathbb{D}_{n}^{\mu}$, we see that

$$
\begin{aligned}
& \left(\mathcal{U}^{-1}\left(x_{1}, \ldots, x_{N}, f\right) \mathbf{H}_{0}^{N} \mathcal{U}\left(x_{1}, \ldots, x_{N}, f\right) \Phi, \Psi\right)_{\mathcal{F}} \\
= & \lim _{N, M \rightarrow \infty}\left(\mathbf{H}_{0}^{N} \sum_{n=0}^{N} \frac{1}{n !}(i \tilde{\Pi}(\hat{f}))^{n} \Phi, \sum_{M=0}^{M} \frac{1}{m !}(i \tilde{\Pi}(\hat{f}))^{m} \Psi\right)_{\mathcal{F}} \\
= & \lim _{N, M \rightarrow \infty}\left(\sum_{n=1}^{N} \sum_{j=0}^{n-1} \frac{(i \tilde{\Pi}(\hat{f}))^{j}\left[\mathbf{H}_{0}^{N}, i \tilde{\Pi}(\hat{f})\right](i \tilde{\Pi}(\hat{f}))^{n-j-1}}{n !} \Phi+\sum_{n=0}^{N} \frac{(i \tilde{\Pi}(\hat{f}))^{n}}{n !} \mathbf{H}_{0}^{N} \Phi,\right. \\
& \left.\sum_{m=0}^{M} \frac{1}{m !}(i \tilde{\Pi}(\hat{f}))^{m} \Psi\right)_{\mathcal{F}}
\end{aligned}
$$

Note that on $C^{\infty}\left(\mathbf{H}_{0}^{N}\right)$

$$
\begin{aligned}
{\left[\left[\mathbf{H}_{0}^{N}, i \tilde{\Pi}(\hat{f})\right], i \tilde{\Pi}(\hat{f})\right] } & =\left[-\sum_{j=1}^{N} A\left(x_{j}, \hat{f}\right), i \tilde{\Pi}(\hat{f})\right] \\
& =\left\|\frac{\hat{f}}{\omega_{\mu}} \sum_{j=1}^{N} e^{-i \cdot x_{j}}\right\|_{L^{2}\left(\mathbb{R}^{d}\right)}^{2}
\end{aligned}
$$

Then the right hand side (r.h.s.) of (3.5) is as follows:

$$
\begin{aligned}
\lim _{N, M \rightarrow \infty} & \left(-V_{e f f}\left(x_{1}, \ldots, x_{N}, f\right) \sum_{n=0}^{N-2} \frac{(i \tilde{\Pi}(\hat{f}))^{n}}{n !} \Phi-\sum_{n=0}^{N-1} \frac{(i \tilde{\Pi}(\hat{f}))^{n}}{n !} \sum_{j=1}^{N} A\left(x_{j}, \hat{f}\right) \Phi\right. \\
& \left.+\sum_{n=0}^{N} \frac{(i \tilde{\Pi}(\hat{f}))^{n}}{n !} \mathbf{H}_{0}^{N} \Phi, \sum_{m=0}^{M} \frac{(i \tilde{\Pi}(\hat{f}))^{m}}{m !} \Psi\right)_{\mathcal{F}} \\
= & \left(\mathbf{H}_{0}^{N} \Phi-\sum_{j=1}^{N} A\left(x_{j}, \hat{f}\right) \Phi-V_{e f f}\left(x_{1}, \ldots, x_{N}, f\right) \Phi, \Psi\right)_{\mathcal{F}} .
\end{aligned}
$$

Since $C^{\infty}\left(\mathbf{H}_{0}^{N}\right) \cap \mathcal{F}^{\infty}$ is dense in $\mathcal{F},(3.4)$ holds on $C^{\infty}\left(\mathbf{H}_{0}^{N}\right) \cap \mathcal{F}^{\infty}$. By (3.1) and the KatoRellich theorem ([19 Theorem X.12]), one can see that for a real valued function $f$ with $\hat{f} \in \mathbb{D}_{-2}^{\mu} \cap \mathbb{D}_{-1}^{\mu}$ and for each $x_{j} \in \mathbb{R}^{d}, j=1, \ldots, N$, the r.h.s. of (3.4) is self-a.djoint on $D\left(\mathbf{H}_{0}^{N}\right)$ and essentially self-adjoint on $C^{\infty}\left(\mathbf{H}_{0}^{N}\right) \cap \mathcal{F}^{\infty}$. Therefore (3.4) holds on $D\left(\mathbf{H}_{0}^{N}\right)$. For a real valued function $f$ with $\hat{f} \in \mathbb{D}_{-4}^{\mu} \cap \mathbb{D}_{-3}^{\mu} \cap \mathbb{D}_{-2}^{\mu} \cap \mathbb{D}_{-1}^{\mu}$, there exist real valued functions $f_{k}$ with 
$\hat{f}_{k} \in \cap_{n=-4}^{\infty} \mathbb{D}_{n}^{\mu}$ such that

$$
\lim _{k \rightarrow \infty}\left\|\frac{\hat{f}}{\omega_{\mu}^{n}}-\frac{\hat{f}_{k}}{\omega_{\mu}^{n}}\right\|_{L^{2}\left(\mathbb{R}^{d}\right)}=0, \quad n=2, \frac{3}{2}, 1, \frac{1}{2} .
$$

Hence by (3.1) and (3.2), it follows that for $\Phi \in D\left(\mathbf{H}_{0}^{N}\right)$,

$$
\begin{aligned}
& s-\lim _{k \rightarrow \infty} \sum_{j=1}^{N} A\left(x_{j}, \hat{f}_{k}\right) \Phi=\sum_{j=1}^{N} A\left(x_{j}, \hat{f}\right) \Phi, \\
& s-\lim _{k \rightarrow \infty} \mathcal{U}\left(x_{1}, \ldots, x_{N}, f_{k}\right) \Phi=\mathcal{U}\left(x_{1}, \ldots, x_{N}, f\right) \Phi .
\end{aligned}
$$

Hence (3.4) holds for a real valued function $f$ with $\hat{f} \in \mathbb{D}_{-4}^{\mu} \cap \mathbb{D}_{-3}^{\mu} \cap \mathbb{D}_{-2}^{\mu} \cap \mathbb{D}_{-3}^{\mu}$.

\subsection{DIAMAGNETIC INEQUALITY}

In this subsection we define a self-adjoint Hamiltonian for the Nelson model and derive diamagnetic inequalities. Let $\mathcal{N}=L^{2}\left(\mathbb{R}^{d N}\right) \otimes \mathcal{F}$. The Hilbert space $\mathcal{N}$ denotes the set of the state vectors of the system of $\mathrm{N}$-nonrelativistic particles interacting with a scalar field. Suppose that $\hat{\rho} \in \mathbb{D}_{-1}^{\mu}$. Then we can define

$$
\mathbf{H}_{I}(\rho)=\int_{\mathbb{R}^{d N}}^{\oplus} \sum_{j=1}^{N} A\left(x_{j}, \hat{\rho}\right) d x_{1} \ldots d x_{N} .
$$

Let $D_{j, \nu},(j=1, \ldots, N, \nu=1, \ldots, d)$, be the generalized $L^{2}$-derivative in the $\nu$-th direction. Put $\sum_{\nu} D_{j, \nu} D_{j, \nu}=\Delta_{j}, j=1, \ldots, N$. The Hamiltonian for the Nelson model with an ultraviolet cut-off function $\rho$ with $\hat{\rho} \in \mathbb{D}_{-1}^{\mu}$ is formally defined as an operator acting in $\mathcal{N}$ as follows:

$$
\mathbf{H}_{\rho}^{N}=-\frac{1}{2} \sum_{j=1}^{N} \Delta_{j} \otimes I-g \mathbf{H}_{I}(\rho)+I \otimes \mathbf{H}_{0}^{N},
$$

where $g$ is a real coupling constant. It is well known that

$$
\mathbf{H}^{N}=-\frac{1}{2} \sum_{j=1}^{N} \Delta_{j} \otimes I+I \otimes \mathbf{H}_{0}^{N}
$$

is self-adjoint on

$$
\mathcal{D}=D\left(-\sum_{j=1}^{N} \Delta_{j} \otimes I\right) \cap D\left(I \otimes \mathbf{H}_{0}^{N}\right) .
$$

The following theorem is well known; 
Theorem 3.2 ([2]) Let $\rho$ be a real valued function with $\hat{\rho} \in \mathbb{D}_{-2}^{\mu} \cap \mathbb{D}_{-1}^{\mu}$. Then $\mathbf{H}_{\rho}^{N}$ is selfadjoint on $\mathcal{D}$ and bounded from below. Moreover; it is essentially self-adjoint on any core of $\mathbf{H}^{N}$.

Let

$$
\mathcal{U}(f)=\int_{\mathbb{R}^{d N}}^{\oplus} \mathcal{U}\left(x_{1}, \ldots, x_{N}, f\right) d x_{1} \ldots d x_{N}
$$

The multiplication operator $V_{e f f}\left(x_{1}, \ldots, x_{N}, f\right)$ is denoted by $V_{e f f}(f)$. Since $V_{e f f}(f)$ is a bounded function,

$$
I \otimes \mathbf{H}_{0}^{N}+g V_{e f f}(f) \otimes I
$$

is self-adjoint on $D\left(I \otimes \mathbf{H}_{0}^{N}\right)$. Similarly to Theorem 3.2, for a real valued function $\rho$ with $\hat{\rho} \in \mathbb{D}_{-2}^{\mu} \cap \mathbb{D}_{-1}^{\mu}$, one can see that

$$
I \otimes \mathbf{H}_{0}^{N}-g \mathbf{H}_{I}(\rho)
$$

is self-adjoint on $D\left(I \otimes \mathbf{H}_{0}^{N}\right)$. The following theorem follows from Lemma 3.1.

Theorem 3.3 Let $\rho$ be a real valued function with $\hat{\rho} \in \mathbb{D}_{-4}^{\mu} \cap \mathbb{D}_{-3}^{\mu} \cap \mathbb{D}_{-2}^{\mu} \cap \mathbb{D}_{-1}^{\mu}$. Then $\mathcal{U}(g \rho)$ maps $D\left(I \otimes \mathbf{H}_{0}^{N}\right)$ onto itself with

$$
\mathcal{U}(g \rho)\left(I \otimes \mathbf{H}_{0}^{N}-g \mathbf{H}_{I}(\rho)\right) \mathcal{U}^{-1}(g \rho)=I \otimes \mathbf{H}_{0}^{N}+g V_{e f f}(\rho) \otimes I
$$

Theorem 3.4 (Diamagnetic Inequality) Let $|V|$ be a multiplication operator which is $-\frac{1}{2} \Delta$ form bounded with relative bound $\epsilon$ and $\rho$ be a real valued function with $\hat{\rho} \in \mathbb{D}_{-2}^{\mu} \cap \mathbb{D}_{-1}^{\mu}$. Then 
$|V|$ is $\mathbf{H}_{\rho}^{N}$-form bounded with the relative bound at most $\epsilon$. Morcover for $V \in P$ the following inequality holds;

$$
\begin{aligned}
& \left|\left(F, e^{-t\left(\mathbf{H}_{\rho}^{N} \dot{+} V_{+} \dot{-} V_{-}\right)} G\right)_{\mathcal{N}}\right| \\
& \leq\left(\|F\|_{\mathcal{F}}, e^{-t\left(-\frac{1}{2} \sum_{j=1}^{N} \Delta_{j}+g V_{e f f} \dot{+} V_{+} \dot{-} V_{-}\right)}\|G\|_{\mathcal{F}}\right)_{L^{2}\left(\mathbb{R}^{d N}\right)}
\end{aligned}
$$

Proof: As in Theorem 2.3, by Theorem 3.3 and the strong Trotter product formula, we have for $V \in L^{\infty}\left(\mathbb{R}^{d}\right)$ and a real valued function $\rho$ with $\hat{\rho} \in \mathbb{D}_{-4}^{\mu} \cap \mathbb{D}_{-3}^{\mu} \cap \mathbb{D}_{-2}^{\mu} \cap \mathbb{D}_{-1}^{\mu}$

$$
\begin{aligned}
& \left|\left(F, e^{-t\left(\mathbf{H}_{\rho}^{N}+V \otimes I\right)} G\right)_{\mathcal{N}}\right| \\
& =\left|\left(F,\left(e^{-\frac{t}{n}\left(-\frac{1}{2} \sum_{j=1}^{N} \Delta_{j} \otimes I\right)} \mathcal{U}(g \rho)^{-1} e^{-\frac{t}{n}\left(I \otimes \mathbf{H}_{0}^{N}+g V_{e f f}(\rho) \otimes I\right)} \mathcal{U}(g \rho) e^{-\frac{t}{n} V \otimes I}\right)^{n} G\right)_{\mathcal{N}}\right| \\
& \leq\left(\|F\|_{\mathcal{F}}, e^{-t\left(-\frac{1}{2} \sum_{j=1}^{N} \Delta_{j}+g V_{e f f}+V\right)}\|G\|_{\mathcal{F}}\right)_{L^{2}\left(\mathbb{R}^{d N}\right)}
\end{aligned}
$$

For any real valued function $\rho$ with $\hat{\rho} \in \mathbb{D}_{-2}^{\mu} \cap \mathbb{D}_{-1}^{\mu}$, there exists a real valued function $\rho_{k}$ with $\hat{\rho}_{k} \in \mathbb{D}_{-4}^{\mu} \cap \mathbb{D}_{-3}^{\mu} \cap \mathbb{D}_{-2}^{\mu} \cap \mathbb{D}_{-1}^{\mu}$ such that for any $\Phi \in \mathcal{D}$

$$
s-\lim _{k \rightarrow \infty} \mathbf{H}_{\rho_{k}}^{N} \Phi+V \Phi=\mathbf{H}_{\rho} \Phi+V \Phi .
$$

Hence, by [18, Theorem VIII.25], $\mathbf{H}_{\rho_{k}}^{N} \rightarrow \mathbf{H}_{\rho}^{N}$ in the strong resolvent sense, which implies that

$$
s-\lim _{k \rightarrow \infty} e^{-t\left(\mathbf{H}_{\rho_{k}}^{N}+V\right)}=e^{-t\left(\mathbf{H}_{\rho}^{N}+V\right)} .
$$

Then (3.6) holds for $\hat{\rho} \in \mathbb{D}_{-2}^{\mu} \cap \mathbb{D}_{-1}^{\mu}$. By the similar limiting argument for $V$ as in Theorem 2.6 , we get the desired results.

By Theorem 3.4, we get the following corollaries.

Corollary 3.5 Let $\rho$ be a real valued function with $\hat{\rho} \in \mathbb{D}_{-2}^{\mu} \cap \mathbb{D}_{-1}^{\mu}$ and $V \in P$. Then

$$
\inf \sigma\left(-\frac{1}{2} \sum_{j=1}^{N} \Delta_{j}+g V_{e f f}(\rho) \dot{+} V_{+}-V_{-}\right) \leq \inf \sigma\left(\mathbf{H}_{\rho}^{N} \dot{+} V_{+} \dot{-} V_{-}\right) \text {. }
$$


Corollary 3.6 (Abstract Kato' inequality) Let $\rho$ be a real valued function with $\hat{\rho} \in \mathbb{D}_{-2}^{\mu} \cap \mathbb{L}_{-1}^{\prime \prime}$ and $V \in P$.

Suppose $\psi \in D\left(\left(-\frac{1}{2} \sum_{j=1}^{N} \Delta_{j}+g V_{e f f}(\rho) \dot{+} V_{+} \dot{-} V_{-}\right)^{\frac{1}{2}}\right), \psi \geq 0$ and $G \in D\left(\mathbf{H}_{\rho} \dot{+} V_{+} \dot{-} V_{-}\right)$. Then $\|G(\cdot)\|_{\mathcal{F}} \in D\left(\left(-\frac{1}{2} \sum_{j=1}^{N} \Delta_{j}+g V_{e f f}(\rho) \dot{+} V_{+} \dot{-} V_{-}\right)^{\frac{1}{2}}\right)$ and the following incquality holds;

$$
\begin{aligned}
\Re( & \left.(\operatorname{sign} G) \psi,\left(\mathbf{H}_{\rho} \dot{+} V_{+} \dot{-} V_{-}\right) G\right)_{\mathcal{N}} \\
\geq & \left(\left(-\frac{1}{2} \sum_{j=1}^{N} \Delta_{j}+g V_{e f f}(\rho) \dot{+} V_{+} \dot{-} V_{-}\right)^{\frac{1}{2}} \psi\right. \\
& \left.\left(-\frac{1}{2} \sum_{j=1}^{N} \Delta_{j}+g V_{e f f}(\rho) \dot{+} V_{+} \dot{-} V_{-}\right)^{\frac{1}{2}}\|G\|_{\mathcal{F}}\right)_{L^{2}\left(\mathbb{R}^{d N}\right)} .
\end{aligned}
$$

\section{REMARKS}

(1) In $[11,13,14]$, in place of $\mathbf{H}_{\rho}^{P}$ defined in PART I, the Hamiltonian with the dipole approximation $\mathbf{H}_{\rho}^{D}$ is considered;

$$
\mathbf{H}_{\rho}^{D}=\frac{1}{2} \sum_{\mu=1}^{d}\left(-i D_{\mu} \otimes I-A_{\mu}^{D}(\hat{\rho})\right)^{2}+I \otimes \mathbf{H}_{0}^{P},
$$

where

$$
A_{\mu}^{D}(\hat{\rho})=\int_{\mathbb{R}^{d}}^{\oplus} A_{\mu}(0, \hat{\rho}) d x
$$

The Hamiltonian $\mathbf{H}_{\rho}^{D}$ can be presented by the constant fiber direct integral

$$
\mathbf{H}_{\rho}^{D}=\int_{\mathbb{R}^{d}}^{\oplus} \mathbf{H}_{\rho}^{D}(p) d p
$$

where

$$
\mathbf{H}_{\rho}^{D}(p)=\frac{1}{2} \sum_{\mu=1}^{d}\left(p_{\mu}-A_{\mu}(0, \hat{\rho})\right)^{2}+\mathbf{H}_{0}^{P}
$$


For a real valued function $\rho$ with $\hat{\rho} \in \mathbb{D}_{-2} \cap \mathbb{D}_{-1}$, it is well known that $\mathbf{H}_{\rho}^{D}$ is self-adjoint on $D(\Delta \otimes I) \cap D\left(I \otimes \mathbf{H}_{0}^{P}\right)\left(\left[9\right.\right.$, Theorem 2.1]) and $\mathbf{H}_{\rho}^{D}(p)$ on $D\left(\mathbf{H}_{0}^{N}\right)([9$, Theorem 2.2]). Moreover suppose that $\rho$ is a real valued function with $\hat{\rho} \in \mathbb{D}_{-3} \cap \mathbb{D}_{-2} \cap \mathbb{D}_{-1}$ and that $\hat{\rho}$ is rotation invariant: $\hat{\rho}(k)=\hat{\rho}(|k|)$. Then there exists a unitary operator $T(p)$ on $\mathcal{F}(\mathcal{W})$ such that

$$
T(p) \mathbf{H}_{\rho}^{D}(p) T(p)^{-1}=\frac{(1-\Lambda)^{2}}{2} p^{2}+\mathbf{H}_{0}^{P}+\eta
$$

where $\Lambda$ and $\eta$ are real constants $([9,10,11])$. Define a unitary operator on $L^{2}\left(\mathbb{R}^{d}\right) \circlearrowleft \mathcal{F}(\mathcal{W})$ by

$$
T=\int_{\mathbb{R}^{d}}^{\oplus} T(p) d p
$$

Then $([9,10,11])$

$$
T \mathbf{H}_{\rho}^{D} T^{-1}=-\frac{(1-\Lambda)^{2}}{2} \Delta \otimes I+I \otimes \mathbf{H}_{0}^{P}+\eta .
$$

In general, however, using the unitary equivalence (4.1), we can not derive the diamagnetic inequality for $\mathbf{H}_{\rho}^{D}+V$. The reason is that the unitary operator $T$ is defined via the constant fiber direct integral on momentum space. Then for each $x \in \mathbb{R}^{d}$ we can not obtain (2.7).

(2) The self-adjoint Hamiltonian considered in [12] has the same action as that of $\mathbf{H}_{\rho}^{P}$ on some domain $\mathcal{D}$, for example, $\mathcal{D}=C_{0}^{\infty}\left(\mathbb{R}^{d}\right) \hat{\otimes} \mathcal{F}^{\infty}(\mathcal{W})$. However essential self-adjointness of $\mathbf{H}_{\rho}^{P}$ is not known. Then one can not mention explicit equality on these Hamiltonians.

(3) In [16], the author introduced the following scaling Hamiltonian

$$
\mathbf{H}_{\rho, \alpha}^{N}=-\frac{1}{2} \sum_{j=1}^{N} \Delta_{j} \otimes I+\alpha \mathbf{H}_{I}(\rho)+\alpha^{2} I \otimes \mathbf{H}_{0}^{N} .
$$

Using a functional integral method, for a real valued function $\rho$ with $\hat{\rho} \in \mathbb{D}_{-2}^{\mu} \cap \mathbb{L}_{-1}^{\mu}$ and $V \in P$, the author obtained the following ([16, Theorem 4.1]);

$$
s-\lim _{\alpha \rightarrow \infty} e^{-t\left(\mathbf{H}_{\rho, \alpha}^{N} \dot{+} V_{+} \dot{-} V_{-}\right)}=e^{-t\left(-\frac{1}{2} \sum_{j=1}^{N} \Delta_{j}+g V_{e f f}(\rho)+V_{+} \dot{-} v_{-}\right)} \oslash \mathbf{P}
$$

where $\mathbf{P}$ denotes the projection onto the vacuum subspace $\{c \Omega \mid c \in \mathbb{C}\}$ in $\mathcal{F}$. Furthermore

$$
\lim _{\alpha \rightarrow \infty} \inf \sigma\left(\mathbf{H}_{\rho, \alpha}^{N} \dot{+} V_{+} \dot{-} V_{-}\right)=\inf \sigma\left(-\frac{1}{2} \sum_{j=1}^{N} \Delta_{j}+g V_{e f f}(\rho)+V_{+} \dot{-} V_{-}\right) \text {. }
$$


TeX-master: $\mathrm{t}$

\section{REFERENCES}

[1] T.A.Welton, Some observable effects of the quantum-mechanical fluctuations of the electromagnetic field, Phys.Rev.74(1948),1157-1167.

[2] E.Nelson, Interaction of nonrelativistic particles with a quantized scalar field, J.Math.Phys.5(1964),1190-1197.

[3] B.Simon, Maximal and Schrödinger forms, J.Operator Theory 1(1979),37-47.

[4] B.Simon, Functional integral and quantum physics, Academic Press(1979).

[5] B.Simon, An abstract Kato's inequality for generator of positivity preserving semigroups, Indiana Univ.Math.J.26(1977),1067-1073.

[6] B.Simon, Kato's inequality and the comparison of semigroup,J.Func.Anal.32 (1979).

[7] H.Hess, R.Schrader, and D.A.Uhlenbrock, Domain of semigroup and generalization of Kato's inequality, Duke Math. J. 44(1977),893-904.

[8] A.Arai, Rigorous theory of spectra and radiation for a model in quantum electrodynamics, J.Math.Phys.24(1983),1896-1910

[9] A.Arai, A note on scattering theory in non-relativistic quantum electrodynamics,J.Phys A:Math.Gen.16(1983),49-70.

[10] A.Arai, An asymptotic analysis and its application to the nonrelativistic limit of the Pauli-Fierz and a spin-boson model, J.Math.Phys.31(1990),2653-2663

[11] F.Hiroshima, Scaling limit of a model in quantum electrodynamics, J.Math.Phys.34 $(1993), 4478-4518$.

[12] F.Hiroshima, Functional integral representation of a model in QED. submitted in J.Func.Anal.

[13] T.Okamoto and K.Yajima, Complex scaling technique in non-relativistic massive QED, Ann. Int. Henri. Poincaré42(1985),311-327 
[14] P.Blanchard,Discussion mathématique du modéle de Pauli et Fierz relatif á la catastrophe intarouge, Comm.Math,Phys.15(1969),156-172.

[15] J.T.Cannon, Quantum field theoretic properties of a model of Nelson: domain and eigenvector stability for perturbed linear operators, J.Func.Anal.8(1971),102-152.

[16] A.Arai,Scaling limit for quantum systems of nonrelativistic particles interacting with a bose field, Hokkaido Univ.preprint series in math. 59 .

[17] F.A.Berezin, Method of second quantization, Academic Press, New York(1966).

[18] M.Reed,B.Simon, Method of modern mathematical physics I, Academic Press, New York(1975).

[19] M.Reed,B.Simon, Method of modern mathematical physics II, Academic Press, New York(1975).

[20] T.Kato, K.Masuda, Trotter's product formula for nonlinear semigroup generated by the subdifferentiables of convex functionals, J.Math.Soc.Japan 30(1978),169-178. 